\title{
Bologna Digital - die digitale Transformation im Europäischen Hochschulraum gestalten
}

\section{Florian Rampelt, Dominic Orr, Alexander Knoth und Renata Suter}

\section{Zusammenfassung}

Die digitale Transformation hat Auswirkungen auf die unterschiedlichsten Bereiche von Studium und Lehre. Dies gilt in Zeiten der COVID-19-Pandemie umso mehr auch für die Auseinandersetzung über Zukunftsperspektiven der Hochschulbildung im europäischen Kontext. Der Beitrag analysiert die Digitalisierung als eine wichtige Aufgabe für den Europäischen Hochschulraum und zeigt zentrale Aktionsfelder für eine Gestaltung der digitalen Transformation im Rahmen des Bologna-Prozesses und der europäischen Zusammenarbeit in der Hochschulbildung auf. Er diskutiert ausgewählte Schwerpunktbereiche mit einer Perspektive auf den Status quo und mögliche Zukunftsszenarien. Fokussiert wird dabei auf Mobilität und Austausch, die Vermittlung von Kompetenzen sowie die Qualitätssicherung als Kernelemente der europäischen Zusammenarbeit.

\author{
F. Rampelt ( $\square)$ \\ Stifterverband, Hochschulforum Digitalisierung, Berlin, Deutschland \\ E-Mail: Florian.Rampelt@stifterverband.de \\ D. Orr \\ Nova Gorica University, Vipava, Slowenien \\ E-Mail: dominic.orr@giz.de
}

\author{
A. Knoth \\ Deutscher Akademischer Austauschdienst - DAAD, Berlin, Deutschland \\ E-Mail: knoth@daad.de \\ R. Suter \\ Kiron Open Higher Education gGmbH, Berlin, Deutschland \\ E-Mail: renata.suter@kiron.ngo
}




\title{
Schlüsselwörter
}

Bologna-Prozess • Europäischer Hochschulraum • Digitalisierung •

Kompetenzen • Mobilität• Qualitätssicherung • Micro-Credentials

\section{Digitalisierung als strategische Aufgabe im Europäischen Hochschulraum}

\begin{abstract}
„Digitalisation plays a role in all areas of society and we recognise its potential to transform how higher education is delivered and how people learn at different stages of their lives. We call on our higher education institutions to prepare their students and support their teachers to act creatively in a digitalised environment" (Paris Communiqué 2018, S. 3).
\end{abstract}

Die Digitalisierung in Bildung, Wissenschaft, Wirtschaft und Gesellschaft hat signifikante Auswirkungen auf Fragen einer zukunftsfähigen Hochschulbildung. Die Auseinandersetzung mit digitalen Technologien und Digitalisierungsprozessen in Studium und Lehre ist daher seit Jahren auf unterschiedlichen politischen Ebenen zu beobachten. Zahlreiche Bundesländer, die Kultusministerkonferenz (KMK 2019) und auch die Europäische Kommission (Europäische Kommission 2018, 2020) haben sich im Rahmen von Strategien und Aktionsplänen mit Fragen der Digitalisierung im Bildungsbereich auseinandergesetzt. 2020 hat die digitale Transformation im Hochschulbereich aufgrund der Folgen der COVID19-Pandemie noch einmal an Bedeutung gewonnen. Hochschulen standen überall vor der Aufgabe, das Studium im Jahr 2020 vorwiegend digital zu gestalten. Gerade auch die für den europäischen Austausch so bedeutsame internationale Mobilität, die stark durch den persönlichen Austausch geprägt ist, konnte kaum mehr wahrgenommen werden (Kercher und Plasa 2020). Bildung in Europa erlebte im Sommer 2020 eine nie zuvor gekannte Disruption, die auch europäische Antworten erfordert: „Due to the COVID-19 pandemic, we witnessed the largest disruption to education and training in Europe's recent history" (Europäische Kommission 2020).

Die Digitalisierung sollte aber nicht nur als Ergebnis einer akuten Disruption verstanden werden, sondern als ein übergreifender, langfristiger Transformationsprozess, der auch unabhängig von COVID-19 zu signifikanten Veränderungen und Anpassungsnotwendigkeiten in allen Leistungsbereichen und Aktivitäten von Hochschulen führt (Kerres 2016). Hochschulleitungen messen diesem Transformationsprozess, der neben der Notwendigkeit einer Entwicklung von klaren Prioritäten und Strategien (Rampelt und Wagner 2020) auch einen politischen 
Handlungsbedarf mit sich bringt, eine zunehmende Bedeutung zu. Um Lösungen für zentrale Herausforderungen der Hochschulbildung zu erarbeiten, müssen in vielen Bereichen die notwendigen Rahmenbedingungen auch über Landesgrenzen hinaus gestaltet werden. An dieser länderübergreifenden Umsetzung mangelt es jedoch der Hochschulbildung oft noch (Gaebel und Zhang 2018).

Die Aufgabe einer zukunftsfähigen strategischen Gestaltung von Hochschulbildung könnte einmal mehr die Rolle Europas in den Mittelpunkt stellen. In den vergangenen 20 Jahren hat die europäische Hochschulpolitik bereits zu signifikanten Veränderungen im Hochschulbereich geführt. Im Rahmen des Bologna-Prozesses wurde seit den späten 1990er-Jahren eine europäische Studienreform initiiert und national umgesetzt, die in ihrer Reichweite unvergleichlich ist. Das Bachelor- und Mastersystem hat Einzug in beinahe jede Hochschule gefunden und Creditpoints gelten als maßgeblich für die Entwicklung von Curricula auf der Grundlage von Workload und Learning Outcomes. Diese Maßnahmen zur Harmonisierung wurden durch Initiativen und Vereinbarungen auf europäischer Ebene erreicht. Doch wie steht es mit den Möglichkeiten einer europäischen Hochschulbildung im digitalen Zeitalter? Welche Rolle spielt die Digitalisierung im Europäischen Hochschulraum der Gegenwart und im Kontext einer europäischen Hochschulbildung der Zukunft?

Der vorliegende Beitrag analysiert die bisherige Auseinandersetzung mit dem Thema im europäischen Kontext ${ }^{1}$ und diskutiert darauf aufbauend zentrale Aktionsfelder der Digitalisierung in Studium und Lehre als strategische Gemeinschaftsaufgabe im Europäischen Hochschulraum. Ein besonderer Fokus wird dabei in Anlehnung an den Schwerpunkt des Sammelbandes auf die Perspektive Deutschlands gelegt. Abschließend werden für die gewählten Aktionsfelder Vorschläge formuliert, wie die Digitalisierung im Europäischen Hochschulraum noch besser konsolidiert, strategisch fokussiert und kollaborativ ausgestaltet werden könnte.

\section{Der Bologna-Prozess: Welche Relevanz hat die Digitalisierung?}

\subsection{Der Bologna-Prozess}

Der Bologna-Prozess umfasst alle Unterzeichnerstaaten eines gemeinsamen Zielkanons, der sogenannten Bologna Declaration, die von inzwischen 48 Staaten des

${ }^{1}$ Stand: Oktober 2020. 
sogenannten Europäischen Hochschulraums (European Higher Education Area, kurz EHEA) unterzeichnet wurde, der damit deutlich größer als die Europäische Union ist. Die daraus entstandene Kollektivarbeit der Unterzeichnerstaaten soll zentrale Impulse für die hochschulpolitische Arbeit der Mitgliedsländer geben. Die Europäische Kommission, eine zentrale Akteurin im Bologna-Prozess, charakterisiert diesen als einen „Mechanismus zur Förderung der zwischenstaatlichen Zusammenarbeit von [derzeit] 48 europäischen Ländern im Bereich der Hochschulbildung". ${ }^{2}$

Alle drei Jahre wird im Rahmen von Minister*innentreffen ein zuvor gemeinsam vorbereitetes Arbeitsprogramm festgelegt. ${ }^{3}$ Dieser Plan dient der Strukturierung der Arbeit von Arbeitsgruppen mit unterschiedlichen Schwerpunktthemen. Zentral für den Bologna-Prozess ist, dass keine Sanktionen für Länder vorgesehen sind, die sich nicht an das gemeinsam vereinbarte Programm und die in den Kommuniqués formulierten Absichtserklärungen der Bologna-Minister*innen ${ }^{4}$ halten. Ergo können die Minister*innen im Rahmen des Bologna-Prozesses nur „Soft Law Governance“ umsetzen (Harmsen und Deca 2019). Konkret muss ein Großteil der notwendigen formalen Maßnahmen in den Mitgliedsstaaten stattfinden. Politische Maßnahmen und Gesetzgebungsprozesse (zum Beispiel zu Qualifikationen) werden auf Grundlage der europäischen Vereinbarungen im nationalen Kontext umgesetzt, in Ländern wie Deutschland oft sogar eine weitere Gliederungsebene niedriger, in den Bundesländern. Diese Prozesslogik gilt auch für eine Gestaltung der Digitalisierung im Europäischen Hochschulraum.

\subsection{Digitalisierung als Thema im Bologna-Prozess}

Im Rahmen der thematischen und strategischen Schwerpunktsetzungen des Bologna-Prozesses kommt den Kommuniqués der regelmäßig stattfindenden Bologna-Minister*innentreffen sowie den begleitenden „Bologna Process Implementation Reports“ (Europäische Kommission 2018) ${ }^{5}$ eine besondere Bedeutung zu. Die Bologna-Kommuniqués setzen jeweils für die Jahre nach einem solchen

\footnotetext{
${ }^{2}$ Siehe: https://ec.europa.eu/education/policies/higher-education/bologna-process-and-eur opean-higher-education-area_de. Zugegriffen: 30.10.2020.

${ }^{3}$ Alle Arbeitsprogramme seit 1999 sind hier einsehbar: https://ehea.info/pid35261/events-byperiod.html. Zugegriffen: 30.10.2020.

${ }^{4}$ Alle Erklärungen und Kommuniqués seit 1999 sind hier einsehbar: https://ehea.info/pageministerial-declarations-and-communiques. Zugegriffen: 30.10.2020.

${ }^{5}$ Alle Implementation und Stocktaking Reports sind hier einsehbar: https://www.ehea.info/ page-implementation. Zugegriffen: 30.10.2020.
} 
Treffen den strategischen Rahmen, von Prag 2001 bis Rom 2020. Sie enthalten bereits seit dem Prager Kommuniqué Verweise auf neue Technologien und insbesondere notwendige Forschungsaktivitäten dazu. Eine proaktive Auseinandersetzung mit den Möglichkeiten und Grenzen digitaler Technologien in Studium und Lehre wird seit dem Yerevan-Kommuniqué am Rand gefordert: „We will encourage and support higher education institutions and staff [...] in fully exploiting the potential benefits of digital technologies for learning and teaching" (Yerevan Communiqué 2015). Solche Prioritäten werden im EHEA-Kontext durch die Arbeit in der Bologna-Follow-up-Gruppe (BFUG) weiter vorangetrieben. Der „Bologna Process Implementation Report“ (European Commission et al. 2018) sammelt ergänzend Studienergebnisse und Daten und analysierte 2018 auch explizit Entwicklungen im Bereich der Digitalisierung von Studium und Lehre. Zentrale Erkenntnis war dabei, dass es nur in vereinzelten Ländern Europas sichtbare strategische Initiativen gab und dies signifikant gestärkt werden müsste, auch durch Maßnahmen auf europäischer Ebene. Auf dieser Grundlage wurde im Pariser Kommuniqué 2018 der Digitalisierung erstmals eine besondere Priorität eingeräumt (European Commission, EACEA und Eurydice 2020, S. 14).

Dennoch wurde das Thema nicht als eigenständiger Schwerpunkt in das Bologna-Arbeitsprogramm von 2018 bis 2020 aufgenommen - vielmehr sollte es als Querschnittsthema Berücksichtigung finden und in den unterschiedlichen Arbeits- und Beratungsgruppen der BFUG schwerpunktmäßig mitaufgegriffen werden. Ein Zusammenschluss von Akteur*innen, den das Hochschulforum Digitalisierung koordiniert, trieb daher über informelle Austauschprozesse unter dem Slogan „Bologna Digital“6 die Arbeit an dem Thema weiter voran und involvierte dabei einen Großteil der zentralen Bologna-Stakeholder (Orr et al. 2020b). Diese Arbeit konnte über die Advisory Group on Teaching and Learning auch in formale Anhörungen zum Bologna-Prozess miteingebracht werden (BFUG 2020a). ${ }^{7}$

\footnotetext{
${ }^{6}$ „Bologna Digital“ entstand 2018 als informelle Initiative für eine stärkere Auseinandersetzung mit der Digitalisierung im Europäischen Hochschulraum. Organisiert vom HFD sollten Kollaborationsanlässe geschaffen werden, um Bedarf und Prioritäten verschiedener Akteur*innen herauszuarbeiten. Als Ergebnis von internationalen Expert*innenworkshops in Berlin und Wien wurden 2019 das White Paper „Bologna Digital 2020“ veröffentlicht und im Frühjahr 2020 ein Positionspapier zum neuen Aktionsplan für digitale Bildung eingereicht, u. a. mit dem Vorschlag einer Stärkung des Peer Learnings und des europäischen Austauschs von Digitalisierungsinitiativen. Weitere Informationen unter https://hochschulforumdigitalisierung.de/en/news/white-paper-bologna-digital2020-digitalisation-higher-education-europe. Zugegriffen: 30.10.2020.

${ }^{7}$ https://www.ehea.info/Upload/AG2_Learning_Teaching_2_Hearing_2.pdf. Zugegriffen: 30.10.2020.
} 
Ergebnis der Initiative war das White Paper „Bologna Digital 2020“ (Rampelt et al. 2019), welches 2019 veröffentlicht wurde.

Mit Blick auf die COVID-19-Pandemie lässt das Jahr 2020 einen von allen Beteiligten unerwarteten disruptiven Wandel in der Bildungslandschaft Europas erkennen. Digitale Technologien und digitale Bildungsangebote rücken damit noch einmal deutlich stärker in den Fokus. Auch die EHEA-Mitgliedsländer formulieren im Kommuniqué für ihre Konferenz in Rom die Erkenntnis, dass digitale Technologien 2020 oft die einzige Möglichkeit für Bildungssysteme boten, weiter zu funktionieren. Neben einer Unterstützung von Hochschulen bei der Nutzung dieser Technologien sollen digitale Kompetenzen und offen lizenzierte Bildungsressourcen verstärkt fokussiert werden (BFUG Secretariat 2020, Rome Communiqué 2020).

\title{
2.3 Die digitale Transformation als Schwerpunktthema der Europäischen Kommission
}

\begin{abstract}
„Digital transformation is one of the European Commission's priorities as set out in its flagship strategy A Europe fit for the digital age. High quality and inclusive education and training are a key part of reaching this ambition and ensuring that all Europeans are prepared to live and work in the digital age" (European Commission 2020b).
\end{abstract}

Auch wenn der Europäische Hochschulraum sich weit über die Grenzen der Europäischen Union hinaus ausdehnt, ist die Europäische Kommission als Mitglied der BFUG einer der zentralen Stakeholder des Bologna-Prozesses und für zahlreiche Maßnahmen zur operativen Umsetzung von Bologna-Vereinbarungen mitverantwortlich. Die Europäische Kommission betreibt im Vergleich zu vielen nationalstaatlichen Akteur*innen und auch anderen Stakeholdergruppen eine sehr progressive Auseinandersetzung mit der Digitalisierung im Bildungsbereich. Ein Ziel ist dabei, durch ,[w]eitreichende, auf nationale Bildungssysteme bezogene Kooperationen innerhalb der EU [...] die Akzeptanz innovativer Ansätze und digitaler Technologien sowie die Entwicklung digitaler Kompetenzen zu fördern" (Lübcke und Wannemacher 2019). Sie hat, als eine Art Kulminationspunkt zahlreicher vorheriger Aktivitäten, im Jahr 2018 einen Aktionsplan digitale Bildung (Europäische Kommission 2018) veröffentlicht, der zentrale Schwerpunkte für die digitale Bildung über alle Bildungsbereiche hinweg aufzeigt. Im Herbst 2020 wurde, geprägt durch die Erfahrungen im Rahmen der COVID-19 Pandemie, ein neuer Aktionsplan für digitale Bildung veröffentlicht, der in den Jahren 2021-2027 eine „Neuaufstellung des Bildungswesens für das digitale 
Zeitalter“ (Europäische Kommission 2020) ermöglichen soll. Der Aktionsplan verfolgt basierend auf umfassenden Konsultationen mit unterschiedlichsten Stakeholdergruppen (European Commission 2020a, b) zwei strategische Prioritäten: die Förderung der Entwicklung eines leistungsfähigen digitalen Bildungsökosystems und den Ausbau digitaler Kompetenzen für den digitalen Wandel (Europäische Kommission 2020).

Ein Teil der von der Europäischen Kommission, den Arbeits- und Beratungsgruppen des Bologna-Prozesses sowie von informellen Initiativen wie Bologna Digital identifizierten Schwerpunktthemen einer Digitalisierung im Europäischen Hochschulraum soll im Folgenden exemplarisch genauer betrachtet werden.

\section{Zentrale Aktionsfelder der Digitalisierung im Europäischen Hochschulraum: Mobilität, Zukunftskompetenzen und Qualitätssicherung}

Die Digitalisierung im Europäischen Hochschulraum ist durch eine enorme Vielfalt an möglichen Schwerpunktthemen und Aktionsfeldern geprägt (siehe Rampelt et al. 2019). Im Bereich Studium und Lehre können die Auseinandersetzung mit Kompetenzen für das digitale Zeitalter, neue Aufgaben der Qualitätssicherung und die Sicherstellung von Mobilität und Austausch als besonders relevante Aktionsfelder identifiziert werden: Mobilität ist nicht nur für das europäische Projekt seit jeher zentral. Sie steht gleichzeitig seit der COVID-19-Pandemie vor einem besonders disruptiven Transformationsprozess, dem nur durch eine Auseinandersetzung mit Möglichkeiten und Grenzen digitaler Lösungen begegnet werden kann. Auch digitale Kompetenzen sind sowohl Fokus der Maßnahmen zur digitalen Bildung im europäischen Bereich (Europäische Kommission 2018; BFUG 2020a) als auch wichtiger Gegenstand nationalstaatlicher Auseinandersetzungen (KMK 2016, 2019). Als zentrale Voraussetzung für die qualitätsorientierte Vermittlung von Kompetenzen wiederum gilt die Qualitätssicherung, ein Schlüsselelement des Bologna-Prozesses und der Maßnahmen zur Harmonisierung im Europäischen Hochschulraum. Diese drei Aktionsfelder sollen im Folgenden, exemplarisch für eine Vielzahl weiterer Themen, genauer betrachtet werden. 


\subsection{Neue Mobilitätsmuster: Internationaler Austausch und virtuelle Mobilität}

\subsubsection{Mobilität als Kernelement des Europäischen Hochschulraums}

Ein zentraler Aspekt der europäischen Hochschulbildung ist die Auslandsmobilität, der grenzübergreifende Austausch mit anderen Studierenden, Lehrenden und Hochschulmitarbeitenden. Der wechselseitige Austausch und das Eintauchen in europäische Nachbarkulturen sind wichtige Mechanismen, um die soziale Kohäsion in Europa zu stärken und die Internationalisierung der Hochschulbildung voranzutreiben. Die ERASMUS-Initiative und aktuell das Erasmus+-Programm stehen beispielhaft für umfassende Maßnahmen zur Stärkung von Mobilität und Austausch innerhalb und außerhalb des Europäischen Hochschulraums. Die akademische Mobilität, insbesondere von Studierenden, stellt dabei eines der wichtigsten Symbole des europäischen Projekts dar und ist ein Kernelement des Bologna-Prozesses (Gemeinsame Erklärung der Europäischen Bildungsminister 1999).

Das Erasmus+-Programm sowie diverse nationale Initiativen waren bislang sehr erfolgreich in der Förderung physischer Mobilität von Studierenden und Wissenschaftler*innen (Souto-Otero et al. 2019). Für den Bereich Education and Training gilt als europäische Benchmark, dass durchschnittlich wenigstens $20 \%$ der Hochschulabsolvent*innen bis 2020 für eine Zeit im Ausland studiert haben sollen. ${ }^{8}$ Innerhalb der EU werden aber zum Teil noch höhere Mobilitätsziele gesteckt. Der Deutsche Akademische Austauschdienst (DAAD) hat in seiner Strategie 2025 als Ziel formuliert, dass der Hälfte aller Studierenden in Deutschland eine Auslands- oder interkulturelle Erfahrung ermöglicht werden soll. Dabei wird explizit der virtuelle Austausch mitberücksichtigt. ${ }^{9}$

\subsubsection{Virtuelle Austauschformate und neue Mobilitätsmuster}

Sieht man von den COVID-19-bedingten Ad-hoc-Maßnahmen ab, so fällt auf, dass die Hochschulen sich schwertun, Strategien für neue Mobilitätsmuster zu finden. Unter dem Begriff der „Internationalisierung zu Hause“ gilt es, Digitalisierung und Internationalisierung zu verknüpfen. Das Konzept wird definiert als „the purposeful integration of international and intercultural dimensions into the formal and informal curriculum for all students within domestic learning environments“

\footnotetext{
${ }^{8}$ Siehe: https://ec.europa.eu/eurostat/web/education-and-training/eu-benchmarks. Zugegriffen: 30.10 .2020 .

${ }^{9}$ Siehe: https://www.daad.de/de/der-daad/wer-wir-sind/strategie-2025/. Zugegriffen: 30.10.2020.
} 
(Beelen und Jones 2015). In Bezug auf den gesamten Studienverlauf stellt sich die Frage, wie Curricula gestaltet sein können, die Präsenzphasen im Seminarraum mit Onlineaktivitäten integrieren. Wie kann auch in Bezug auf neue Mobilitätsmuster die Teilhabe an unterschiedlichen Bildungsressourcen sowie europäischer Austausch ermöglicht werden? Präsenz- (offline), digital unterstützte (blended) und rein digitale Angebote (online) spielen hier eine Rolle. Diese Vielfalt führt aber auch klar zu größeren didaktischen Unterschieden und Herausforderungen, wie Erfahrungsberichte zeigen (Planells-Artigot und Moll-Lopez 2020).

Umso wichtiger wird es sein, Auslandsmobilität künftig didaktisch fundiert und gleichzeitig systematisch mit digitalen Lehr-Lern-Szenarien zu verzahnen, ohne den analogen Austausch grundsätzlich infrage zu stellen. Erste Analysen zu COVID-19 machen auch übergreifend deutlich, dass sich Verhalten und Interessen internationaler Studieninteressierter verändern, sodass virtuelle Angebote Schlüssel zur Rekrutierung und Betreuung von internationalen Studierenden werden (BridgeU 2020).

\subsubsection{Digitaler Austausch im Europäischen Hochschulraum: Erasmus + und europäische Hochschulnetzwerke}

Die COVID-19-Pandemie hat zur grundlegenden Erosion der physischen europäischen Auslandsmobilität geführt. Das Erasmus+-Programm lief zwar auch 2020 weiter, spätestens ab dem Sommer aber oft virtuell oder in Form von Blended Mobility. Finanzielle Unterstützung wurde dabei trotzdem gewährt und die Nachfrage blieb ungebrochen hoch. ${ }^{10}$ Die Digitalisierung des Erasmus+-Programms ist gleichzeitig eines der großen Themen für die Zukunft der europäischen Hochschulzusammenarbeit. Diverse digitale Aktivitäten werden derzeit unter den Bedingungen der COVID-19-Pandemie getestet, um ab 2021 im gesamten Erasmus+-Programm angewandt und dem Label „Erasmus + Digital“ gerecht werden zu können. So werden etwa Erasmus+-Mobilitätsstipendien digitalisiert, sodass Auslandsaufenthalte digital begonnen und später analog fortgesetzt werden können. ${ }^{11}$ Bedeutsam hierfür sind auch Infrastrukturfragen: Die European Student Card Initiative ${ }^{12}$ bündelt in der neuen Programmgeneration Projekte wie die European Student Card und Erasmus Without Papers, beides zukünftig verpflichtende digitale Infrastrukturvorhaben, welche unter anderem die einheitliche

\footnotetext{
${ }^{10}$ Siehe: https://eu.daad.de/service/erasmus-und-covid-19/de/76954-erasmus-laeuft-im-win tersemester-weiter--wegen-covid-19-erstmals-auch-virtuell/. Zugegriffen: 30.10.2020.

${ }^{11}$ Siehe: https://ec.europa.eu/programmes/erasmus-plus/resources/coronavirus-impact_en. Zugegriffen: 30.10.2020.

${ }^{12}$ Siehe: https://ec.europa.eu/education/education-in-the-eu/european-student-card-initiativ e_en. Zugegriffen: 30.10.2020.
} 
Identifikation von Studierenden, den digitalen Austausch von Mobilitätsdaten und die digitale Administration von Partnerschaftsabkommen zum Ziel haben.

Eine ganz besondere Bedeutung für die europäische Zusammenarbeit im Hochschulbereich haben die sogenannten „Europäischen Hochschulen“. Die seit 2017 diskutierte und seit 2019 umgesetzte Idee stellt eine Leitinitiative der EU zum Aufbau des europäischen Bildungsraums bis 2025 dar. Bis 2020 haben 39 Pilotallianzen die Arbeit aufgenommen. Die Berücksichtigung von Aspekten der Digitalisierung in Studium und Lehre war zu Beginn kein zentrales Kriterium für die Auswahl eines europäischen Hochschulnetzwerks, sondern vielmehr Mittel zum Zweck, das als solches nicht separat herausgehoben wurde. Die Lage änderte sich mit der zweiten Förderkohorte im Jahr 2020, die auch auf die COVID-19Pandemie reagiert. Im hierfür aktualisierten „European Universities Factsheet“ wird dies bereits unter der Rubrik „Why European Universities?“ sehr deutlich:

„The COVID-19 pandemic has suddenly accelerated the digital transformation of higher education institutions. Yet, much more needs to be done for deep technological and structural changes to the benefit of learning and teaching, allowing for more inclusion and flexible learning approaches“ (European Commission 2020c, S. 1).

Damit rücken der durch die Digitalisierung vorangetriebene strukturelle Wandel und insbesondere die Flexibilisierung von Lernpfaden prominent in den Fokus. Um das zu erreichen, sollen die involvierten Hochschulen „digitale und physische Ressourcen in Lehre, Wissen, Daten und Infrastruktur" (Forschung \& Lehre 2020) teilen. Der Aufbau von digitalen, innovativen und standortübergreifenden Infrastrukturen stellt in den meisten Fällen den ersten Schritt hin zur Entwicklung eines gemeinsamen Campus(-Angebots) dar. Ein Beispiel hierfür ist der Virtual Campus des Europäischen Hochschulnetzwerks YUFE (Young Universities for the Future of Europe) an dem aus Deutschland die Universität Bremen und Kiron beteiligt sind. ${ }^{13}$

Insgesamt zeigen sich erste Ansätze einer proaktiven Auseinandersetzung mit digital gestützten und auch rein virtuellen Mobilitäts- und Austauschszenarien auf europäischer Ebene. Sie weisen gleichzeitig auch auf die Notwendigkeit weiterer Impulse und vor allem einer konkreten Umsetzung hin, damit die Digitalisierung sich auch in angepassten Curricula, Programmrichtlinien und Kooperationsvereinbarungen niederschlägt.

${ }^{13}$ Siehe: https://yufe.eu/. Zugegriffen: 30.10.2020. 


\subsection{Kompetenzen für das digitale Zeitalter}

\subsubsection{Digitale Kompetenzen in einem Europa des Wissens}

Bereits in der Bologna-Erklärung hieß es, dass ein „Europa des Wissens“ in der Lage sein sollte, ,seinen Bürgern die notwendigen Kompetenzen für die Herausforderungen des neuen Jahrtausends [...] [zu] vermitteln“ (Gemeinsame Erklärung der Europäischen Bildungsminister 1999). Und auch ,,in der LissabonStrategie [...] adressierte die Kommission die ,Förderung neuer Grundfertigkeiten, insbesondere im Bereich der Informationstechnologien" als eines der zentralen Aufgabenfelder des Hochschulsektors" (Lübcke und Wannemacher 2019). Zwanzig Jahre später gilt dieser Anspruch umso mehr für ein Europa im digitalen Zeitalter. Ein Europa, das fit für das digitale Zeitalter sein will, benötigt eine Gesellschaft, die informiert, reflektiert und kompetent mit digitalen Technologien und deren Anwendung agiert.

„Digitale Kompetenz bedeutet, dass man digitale Technologien souverän und kritisch Nutzen [sic!] kann, und umfasst die Kenntnisse, Fertigkeiten und Einstellungen, die alle Bürgerinnen und Bürger in einer sich rasant verändernden digitalen Gesellschaft brauchen" (Europäische Kommission 2018, S. 8).

Die Fähigkeiten, die Lernende im digitalen Zeitalter benötigen, sind dabei nicht ausschließlich auf Themen der Digitalisierung beschränkt und umfassen neben den digitalen auch nichtdigitale Kompetenzen sowie (fach)spezifische und allgemeinere Fähigkeiten (vgl. Rampelt et al. 2019; Ehlers 2020). Der Kanon all dieser Kompetenzen wird oft unter dem Konzept der „Future Skills“ zusammengefasst (vgl. Ehlers 2020). Diese Kompetenzen lassen sich als Fähigkeiten und Fertigkeiten beschreiben, die ein erfolgreiches Handeln unter den Bedingungen zukünftiger Gesellschaften ermöglichen. Im Kern geht es darum, Lernende von heute auf eine in vielen Bereichen noch ungewisse Zukunft von morgen vorzubereiten.

\subsubsection{Europäische Pionierarbeit im Bereich digitaler Kompetenzen}

Ein Schwerpunkt der Auseinandersetzung mit Zukunftskompetenzen im Europäischen Hochschulraum liegt auf dem Umgang mit digitalen Technologien - gleichermaßen bezogen auf Studierende und auf Lehrende. Die Europäische Kommission hat hier maßgebliche Pionierarbeit geleistet. Das sogenannte „Digital Competence Framework for Citizens“ (DigiComp) ${ }^{14}$ und weitere Qualifikationsrahmen für digitale Kompetenzen dienten als zentraler Referenzrahmen

\footnotetext{
${ }^{14}$ Weitere Informationen unter https://ec.europa.eu/jrc/en/digcomp/digital-competence-fra mework. Zugegriffen: 30.10.2020.
} 
in unterschiedlichen Bildungsbereichen in europäischen Ländern. Auch für die Strategie der deutschen Kultusministerkonferenz (KMK) zur „Bildung in einer digitalen Welt" (KMK 2016) haben solche europäischen Maßnahmen wichtige Impulse gesetzt. Zur Umsetzung dieser Strategie wurde für den Hochschulbereich die Bedeutung digitaler Kompetenzen klar unterstrichen: Im Rahmen eines jeden Studiums sollten sowohl fachspezifische als auch grundlegende digitale Kompetenzen vermittelt und dies unter anderem im Rahmen von Akkreditierungsverfahren geprüft werden. Hier gilt es, auch die grundlegenden Errungenschaften des Bologna-Prozesses wieder aufzugreifen: die Einigung auf gemeinsame, länderübergreifende Rahmenbedingungen und Standards, die auch in Bezug auf digitale Kompetenzen in Hochschulcurricula erreicht werden könnten.

Der digitale Kompetenzrahmen lässt sich aber auch weiterdenken: Ein spezieller Kompetenzrahmen für Lehrende (DigiCompEdu) vereint nationale und regionale Anstrengungen, um die auf die pädagogisch tätigen Personen oder Lehrenden bezogenen digitalen Kompetenzen zu erfassen (Redecker 2017). In den Mitgliedsländern wird er jedoch sehr unterschiedlich umgesetzt und öfter auf den Schul-, aber noch selten auf den Hochschulbereich angewandt. Eine Schnittstellenfunktion könnte hier die Lehrkräftebildung haben.

\subsubsection{Kompetenzen offen und zugänglich vermitteln: von digitalen Lernplattformen zu Open Educational Resources im Hochschulbereich}

Neue Ansätze des Kompetenzerwerbs erfordern sowohl Inhalte und Formate, die dynamisch und kontextbezogen weiterentwickelt werden können, als auch neue Lernprozesse, die unterschiedlichen Kontextbedingungen und den Bedarfen Lernender gerecht werden. Offene Bildungsressourcen (Open Educational Resources; OER) können dabei sowohl als Instrument als auch als Treiber des Prozesses dienen (Orr et al. 2015; Deimann 2020) und zur Förderung von Wissensgesellschaften und Zugang zu Bildung beitragen (UNESCO 2020).

OER sind Bildungsressourcen, die offen lizenziert zur Nachnutzung und Weiterentwicklung (idealerweise im Austausch zwischen Lehrenden und Lernenden) zur Verfügung stehen (Miao et al. 2019; dos Santos et al. 2016). Offene Bildungsangebote lassen sich dabei im übergreifenden Diskurs zur Öffnung von (Hochschul-)Bildung verorten. Offene Bildungsressourcen sind nur ein Teil von „,unterschiedliche[n] Ideen, Konzepte[n] und Ansätze[n], die als Spielarten von Open Education gelten“ (Deimann 2016, S. 17). Deimann (2016) zählt hierzu Open Access, Open Educational Resources, Open Educational Practices, historische Formen von Open Education und Massive Open Online Courses (MOOCs). Auch wenn es vielfach politische Absichtserklärungen dazu gab, scheint das 
Thema Open Education im Allgemeinen sowie OER im Speziellen nach wie vor nicht die zentrale Rolle zu spielen, die möglich wäre. „The contribution of OERs and MOOCs to increase access and improve quality in learning cannot be underestimated" (UNESCO 2020).

Gerade die Entwicklung von MOOCs zeigt dabei aber auch durchaus Gefahren einer „Instrumentalisierung von Open Education“ (ebd., S. 17), die teils geprägt ist durch zunehmend kommerzielle Modelle im (Hochschul-)Bildungsbereich. Plattformen, die den Markt dominieren und vereinzelt auch bereits in deutschen und europäischen Hochschulkontexten eingesetzt werden, stammen oft aus den USA (Coursera, edX, Udacity, vgl. Rampelt et al. 2018) und nicht aus Europa. Es stellt sich dabei die Frage, welche europäischen Lösungen es geben kann, um eigene Daten(sicherheits)standards und übergreifend ,sichere digitale Bildungsräume“ (BMBF 2019) zu gewährleisten. Auf europäischer Ebene wurde mit dem ersten Aktionsplan für digitale Bildung die „Einrichtung einer europaweiten Plattform für die digitale Hochschulbildung" (Europäische Kommission 2018) als zentrale Perspektive formuliert. Bisher hat sich aber keine einzelne Plattform als eine Art europäische Hochschulcloud herauskristallisiert und Varianten einer Förderung der Interoperabilität, Kooperation und Vernetzung bestehender Initiativen scheinen deutlich wahrscheinlicher. ${ }^{15}$

In themenspezifischen Bereichen, wie insbesondere Datenkompetenzen, digitalen Kompetenzen und Kompetenzen im Bereich Künstlicher Intelligenz gibt es, auch aufgrund der Prioritäten der Europäischen Kommission, große Potenziale einer europäischen Zusammenarbeit. So wird seit 2019 vom BMBF mit dem KI-Campus eine Lernplattform für Künstliche Intelligenz gefördert, die im Sommer 2020 als Betaversion deutsch- und englischsprachig online gegangen ist. ${ }^{16}$ Diese Plattform, die auf europäischer Ebene früh auch mit dem Land Luxemburg kooperierte, könnte ein Pilotmodell für durch Open-Source- und OER-Prinzipien geprägte Lösungen mit europäischem Profil darstellen.

Aufgabe der europäischen Stakeholder, der EHEA-Mitgliedsländer und ganz besonders auch der Europäischen Kommission muss es sein, die skizzierten Ansätze zur Stärkung von Kompetenzen für das digitale Zeitalter noch stärker strategisch zu unterstützen und dabei insbesondere Möglichkeiten der Interoperabilität und der Zusammenarbeit zu fördern. Die Offenheit von Bildungsressourcen und Technologien sollte dabei eine zentrale Rolle spielen.

\footnotetext{
${ }^{15}$ Eine Gruppe europäischer Initiativen hat sich etwa im European MOOCs Consortium zusammengeschlossen. Dazu zählen u. a. FUN aus Frankreich, Miríadax aus Spanien und FutureLearn aus Großbritannien. Weitere Informationen unter https://emc.eadtu.eu/emc/par tners. Zugegriffen: 30.10.2020.
}

${ }^{16}$ Siehe: www.ki-campus.org/. Zugegriffen: 30.10.2020. 


\subsection{Qualitätssicherung und Zertifizierung}

\subsubsection{Qualitätssicherung digitaler Lehr- und Lernszenarien}

Die Etablierung der Qualitätssicherung als Schlüsselelement des Hochschulwesens ist eine der Erfolgsgeschichten des Bologna-Prozesses. Im Jahr 1999, als die Bologna-Erklärung unterzeichnet wurde, gab es nur vereinzelt Qualitätssicherungssysteme, mittlerweile gelten sie als Schlüsselelement, um Harmonisierung und qualitätsorientierte Verbesserung von Curricula und Qualifikationen zu ermöglichen und das gegenseitige Vertrauen in Bezug auf Hochschulbildung und Anerkennungsfragen zwischen den EHEA-Mitgliedsstaaten zu stärken (Szabó und Tück 2018). Seit Beginn des Bologna-Prozesses haben 30 Länder ein voll funktionsfähiges Qualitätssicherungssystem für alle Hochschuleinrichtungen eingerichtet, das von im European Quality Assurance Register for Higher Education (EQAR) registrierten Agenturen umgesetzt wird (European Commission, EACEA und Eurydice 2020).

Digitale Lehr- und Lernszenarien sind bisher jedoch oft nicht systemisch hinterlegt und unterliegen hinsichtlich der formalen Anforderungen noch großer Unsicherheit. Einerseits ermöglichen neue Formen von Lernangeboten potenziell flexiblere und stärker personalisierte Lehr- und Lernszenarien und die Transparenz der didaktischen Gestaltung könnte erhöht werden. Andererseits sind grundlegende Fragen zu rechtlichen Aspekten wie Urheberrecht und Datenschutz oft noch offen. Große Herausforderungen ergeben sich etwa im Kontext der Identitätsfeststellung bei digitalen Abschlussprüfungen (vgl. Rampelt et al. 2018). Zusätzlich bringen digitale Lehr- und Lernszenarien auch neue didaktische Anforderungen mit sich. Die COVID-19-Pandemie hat all diese offenen Fragen in Bezug auf die Qualitätssicherung digitaler Lehr- und Lernszenarien dringender denn je gemacht: „A sharp shift to distance and blended education modes also necessitates revisiting $[\ldots]$ quality assurance mechanisms, transparency tools, and recognition procedures" (BFUG 2020b, S. 4).

Im Rahmen des Bologna-Prozesses wurden bereits klare Standards und Richtlinien (u. a. HRK 2015) festgelegt, die grundsätzlich auf digital gestütztes Lernen und Lehren angewendet werden können. Die Qualitätssicherungssysteme und -prozesse müssen jedoch bis zu einem gewissen Grad an neue Lernprozesse und -formate angepasst werden (vgl. Huertas et al. 2018; Rampelt et al. 2018). Dabei sollten bestehende Kriterien und Maßnahmen nicht ersetzt, sondern erneuert und ergänzt werden, um der Digitalisierung in Studium und Lehre angemessen Rechnung zu tragen und Sicherheit und Transparenz für alle Beteiligten zu gewährleisten. Darüber hinaus sollten Qualitätsstandards für digitale Technologien und Daten, die von Hochschulen eingesetzt werden, diskutiert werden, da sie zur 
De-facto-Lernumgebung der Lernenden beitragen. Die COVID-19-Pandemie hat hier, insbesondere durch zahlreiche Ad-hoc-Maßnahmen, oft zu Rechtsunsicherheit, zum Beispiel in Bezug auf den Einsatz kommerzieller Software im Kontext akademischer Lehre und Prüfungen, geführt. Diesen neuen Anforderungen muss mit einer Ausweitung von Standards und Leitlinien begegnet werden.

An der Schnittstelle zwischen dem qualitätsgesicherten Kompetenzerwerb und digitalen Transformationsprozessen kann auch eine Verbindung zwischen der Qualitätssicherung sowie der Anerkennung oder Anrechnung von Kompetenzen (Rampelt et al. 2018) hergestellt werden, die im Rahmen des Bologna-Prozesses traditionell als zwei getrennte (aber miteinander verbundene) Themenbereiche betrachtet wurden, unter einer lernendenzentrierten Perspektive jedoch stärker miteinander verschmelzen. In diesem Zusammenhang wurden in den letzten Jahren neue Methoden und Qualitätsstandards für Qualifizierung, Zertifizierung und Zeugniserteilung diskutiert (Camilleri und Rampelt 2018; Rampelt et al. 2019). Ein besonders aktuelles Konzept stellen dabei sogenannte Micro-Credentials dar.

\subsubsection{Micro-Credentials als Zertifizierungsform zur Förderung neuer Lernwege}

„Another beneficial discovery of the crisis relates to the concept of Micro-Credentials“ (BFUG 2020b).

Bei Hochschulbildung geht es um die Erarbeitung und Vermittlung von Wissen und Kompetenzen, aber auch um die Ausstellung von Zertifikaten für Lernleistungen an Personen, die von Dritten, die nicht Teil des Lernprozesses waren, anerkannt werden. Dies ermöglicht Einzelnen den Übergang von einem Abschnitt des Bildungssystems zum nächsten, von der Hochschulbildung in den Arbeitsmarkt, sowie die Sichtbarmachung erworbener Kompetenzen über die gesamte Bildungs- und Erwerbsbiografie. In der ersten Phase der Bologna-Reformen führten alle Hochschulsysteme eine Struktur von vier Bologna-Zyklen ein, die mit Zertifikaten auf verschiedenen akademischen Niveaus enden. Es handelte sich dabei um eine Harmonisierung der Niveaus und der Nomenklatur, die darauf abzielte, die Anerkennung von Qualifikationsnachweisen system- und länderübergreifend zu erhöhen (Hackl 2001).

Aber ist diese vergleichsweise starre Struktur für die Zukunft der Hochschulbildung zeitgemäß und effektiv? Eine der wesentlichen Einschränkungen der Hochschulbildung in einer sich stetig verändernden Welt besteht darin, dass hohe administrative Hürden für den Zugang zu Lernprogrammen bestehen und diese Programme in der Regel lange dauern (Orr et al. 2020a). Darüber hinaus wird 
bisher als Regelfall angenommen, dass Lernpfade linear und sequenziell verlaufen. Die EUROSTUDENT-Daten für Studierende an europäischen Hochschulen zeigen jedoch, dass viele Studierende ihren Bildungsweg auf dem Weg zum Abschluss unterbrechen (Hauschildt et al. 2018). Und während in Politik und Praxis vom lebenslangen Lernen die Rede ist, erhöhen additive kleinere Lernblöcke während des Lebensweges bisher den Wert des formalen Bildungsprofils einer Person tendenziell nicht. Dies hat zur Konsequenz, dass die Hochschulbildung Personen vorbehalten bleibt, die in der Lage sind, diesen umfassenden Programmen zu folgen, und für die Zeit finanzielle Unterstützung erhalten. Auch wenn die Diskussionen über flexiblere Hochschulbildung bereits seit Langem geführt werden, so ist erst mit der COVID-19-Pandemie, mit unmittelbar unterbrochenen Bildungsverläufen, ein verstärkter Fokus darauf zu beobachten (BFUG 2020b).

Wie kann also europäische Hochschulbildung organisiert werden, sodass sie mehr Inklusivität, Individualisierung und Flexibilität unter den zuvor skizzierten Rahmenbedingungen ermöglicht, zeitgemäßen Kompetenzerwerb sicherstellt und gleichzeitig die zuvor skizzierten Qualitätsanforderungen weiterhin bedeutsam bleiben? Micro-Credentials werden als ein möglicher Weg gesehen, um Flexibilität von Bildungswegen zu ermöglichen, die Anerkennung und Anrechnung verschiedener Lehr- und Lernszenarien zu verbessern, Lernangebote bedarfsorientierter zu gestalten und Kohärenz, Transparenz und Wert für nichtlineare Lernpfade zu schaffen (Chakroun und Keevy 2018; ICDE 2019; Kato et al. 2020). Zwar gibt es bisher keine einheitlichen Definitionen, was genau Micro-Credentials sind, aber die verwendeten Definitionen enthalten oft als gemeinsame Merkmale die begrenzte Dauer der Lernaktivitäten, die zu einem Micro-Credential führen, und die Arbeitsmarktrelevanz der Kompetenzen (Resei et al. 2019).

Hochschulen und insbesondere Anbieter von nichttraditionellen und digitalen Lernangeboten bieten solche Micro-Credentials bereits in unterschiedlichen Ausprägungen für Lernende an. Um jedoch wirklich nutzbar zu werden, müssen Micro-Credentials mindestens die vier folgenden Voraussetzungen erfüllen, die im Rahmen von übergeordneten Rahmenbedingungen sichergestellt werden sollten (vgl. Orr et al. 2020a):

- Transparenz: Micro-Credentials sollten transparent dokumentieren, was Zertifikatsinhabende getan haben, um das Credential zu erlangen, wie dies überprüft wurde und wer den Kompetenzerwerb bestätigt hat.

- Vertrauen: Micro-Credentials sind Teil einer Gruppe von Reformen, die kollektiv als alternative Credentials bezeichnet werden. Entscheidend für deren 
Nutzen ist das Vertrauen in den Prozess zur Verleihung der Zertifikate, da Lernende sonst Dritte von den Kompetenzen weiterhin im Einzelfall überzeugen müssen.

- Flexibilität: Micro-Credentials sollen flexiblere Lernwege erleichtern. Diese sollten sowohl im formalen als auch im nichtformalen Bereich stattfinden.

- Innovation: Insgesamt ergibt sich das Interesse an Micro-Credentials aus ihrem Potenzial, Innovationen bei der Anerkennung und Zertifizierung von Lernprozessen zu erleichtern.

Natürlich können innovative Einzelakteur*innen, Plattformen oder Institutionen jederzeit digitale Zertifikate ausstellen, aber um einen breiten Mehrwert für Empfänger*innen zu sichern, sind kollektive politische Anstrengungen erforderlich. Positiv zu vermerken sind daher aktuelle Entwicklungen auf EHEA-Ebene. Das Projekt MICROBOL (Micro-credentials linked to the Bologna key commitments $)^{17}$ arbeitet mit Akteur*innen des Europäischen Hochschulraums zusammen, um im Rahmen des Bologna-Prozesses zu untersuchen, ob und wie die bestehenden Bologna-Instrumente für Micro-Credentials genutzt werden können (MICROBOL 2020). Dies sind gute Voraussetzungen für eine zielführende Auseinandersetzung mit der Digitalisierung als strategischer Aufgabe im Europäischen Hochschulraum, nämlich über die Erleichterung und Förderung von neuen Lernwegen durch digitale Technologien und innovative Zertifikate unter Berücksichtigung der bisherigen Bologna-Ziele und -Instrumente.

\section{$4 \quad$ Ausblick}

„The COVID-19 pandemic has seen rapid progress in switching to digital learning and teaching. There will undoubtedly be many lessons to learn from the experience [...], but it is clear that a big step forward has been taken and even when a full return to normality is possible, the use of digital technologies will no doubt continue and intensify. [...] There is an important role for strategic policy planning at both national and European levels" (European Commission, EACEA und Eurydice 2020, S. 160 f.).

Die Digitalisierung in Studium und Lehre gewinnt zunehmend an Relevanz im Europäischen Hochschulraum. Durch die COVID-19-Pandemie ist auch auf politischer Ebene eine verstärkte Sensibilität hierfür erkennbar. Ob die Kombination aus gemeinsamen Herausforderungen aufgrund der COVID-19-Pandemie und bereits existierenden oder im Aufbau befindlichen kollektiven Arbeitsstrukturen,

\footnotetext{
${ }^{17}$ Weitere Infos unter https://Microcredentials.eu/. Zugegriffen: 30.10.2020.
} 
etwa im Rahmen des Bologna-Prozesses oder der Europäischen Hochschulnetzwerke, hier die geeigneten Foren und Kollaborationsanlässe für konkrete Ergebnisse auf europäischer und nationaler Ebene bieten, bleibt abzuwarten. Wie eine europäische Zusammenarbeit auf themenspezifischer Ebene ausgestaltet werden kann, haben wir anhand von zentralen Aktionsfeldern der Digitalisierung im Europäischen Hochschulraum aufgezeigt. Die dargestellten Schwerpunkte und Beispiele zeigen exemplarisch nicht nur den Status quo, sondern auch klare Potenziale einer europäischen Auseinandersetzung mit der Digitalisierung in Studium und Lehre auf. Deutlich wird dabei, dass neben der zunehmend erfolgreichen operativen Umsetzung an den Hochschulen vor Ort auch die übergreifende PolicyEbene zur Digitalisierung in Studium und Lehre gestärkt werden muss und auch die Weiterentwicklung von europäischen Förderprogrammen eine wichtige Rolle spielt. Zur weiteren Priorisierung sollen für die drei Aktionsfelder jeweils zwei Kernthesen eine erste Orientierung und mögliche Impulse bieten.

\section{Neue Mobilitätsmuster: Internationaler Austausch und virtuelle Mobilität}

- Digitale Mobilitätsfenster: Flexible und virtuelle Lehr-Lern-Angebote sollten in den Curricula verankert werden. Europäische Programme könnten dies unterstützen.

- Europäische Hochschulnetzwerke sollten mit einem starken digitalen Profil weiter gestärkt und mit einem Fokus auf Teilhabemöglichkeiten ausgebaut werden.

\section{Kompetenzen für das digitale Zeitalter}

- Digitale Kompetenzen, insbesondere Daten- und KI-Kompetenzen, sollten gestärkt und systematisch, auf Grundlage von europäischen Kompetenzrahmen, in Curricula integriert werden.

- Der Europäische Hochschulraum braucht eine OER-Offensive. Offene Bildungsressourcen sollten als Grundlage des Wissens- und Kompetenzerwerbs in Europa gestärkt werden.

\section{Qualitätssicherung und Zertifizierung}

- Bologna-Instrumente sollten weiterentwickelt werden, um systematisch und qualitätsorientiert auf digitale Lehr-Lern-Szenarien angewendet werden zu können.

- Micro-Credentials sollten europaweit als Grundlage für die weitere Auseinandersetzung mit flexiblen Lernpfaden und als Bausteine lebenslangen Lernens gestärkt werden. 


\section{Literatur}

Beelen, J., \& Jones, E. (2015). Defining “internationalisation at home”. University World News. https://www.universityworldnews.com/post.php?story=201512021443 53164. Zugegriffen: 30. Okt. 2020.

Bologna Follow-Up Group (BFUG). (2020a). Advisory group 2 on learning and teaching. Final report. https://ehea.info/Upload/BFUG_DE_UK_73_6_2_AG2_Final_Report.pdf. Zugegriffen: 30. Okt. 2020.

Bologna Follow-Up Group (BFUG). (2020b). Split BFUG meeting: Report from the discussions on the parallel sessions on the implications of COVID-19 for the Bologna process. https://www.ehea.info/Upload/Board_DE_UK_72_5_1_Report_parallel_sessions_ Covid_19.pdf. Zugegriffen: 30. Okt. 2020.

BFUG Secretariat. (2020). Draft 6 of the Rome Ministerial Communiqué, 10 September 2020. https://ehea.info/Upload/BFUG_DE_UK_73_9_Draft_6_Rome_Commun ique.pdf. Zugegriffen: 30. Okt. 2020.

BridgeU. (2020). How will COVID-19 really impact international students' enrolments in 2020 \& 2021? An in-depth analysis of what is affecting students' decisions in 2020 \& what the future holds for 2021. London: BridgeU. https://universities.bridge-u.com/blog/ resources/covid-report/. Zugegriffen: 30. Okt. 2020.

Bundesministerium für Bildung und Forschung (BMBF). (2019). Digitale Zukunft: Lernen. Forschen. Wissen. Die Digitalstrategie des BMBF. Berlin. https://www.bmbf.de/upload_ filestore/pub/BMBF_Digitalstrategie.pdf. Zugegriffen: 30. Okt. 2020.

Camilleri, A., \& Rampelt, F. (2018). Assuring the quality of credentials to support learning innovation. Vienna: 13th European Quality Assurance Forum: Broadening the Scope of QA. https://www.researchgate.net/publication/329059732_Assuring_the_Qua lity_of_Credentials_to_support_Learning_Innovation. Zugegriffen: 30. Okt. 2020.

Chakroun, B., \& Keevy, J. (2018). Digital credentialing - Implications for the recognition of learning across Borders. Paris: UNESCO. https://unesdoc.unesco.org/ark:/48223/pf0000 264428. Zugegriffen: 30. Okt. 2020.

Deimann, M. (2016). Open Education - Die ewig Unvollendete. Synergie. Fachmagazin für Digitalisierung in der Lehre, 2, 14-19. https://www.synergie.uni-hamburg.de/de/media/ ausgabe02/synergie02.pdf. Zugegriffen: 30. Okt. 2020.

Deimann, M. (2020). Lernen mit Open Educational Resources. In H. Niegemann \& A. Weinberger (Hrsg.), Handbuch Bildungstechnologie (S. 699-708). Berlin: Springer.

Ehlers, U.-D. (2020). Future Skills. Lernen der Zukunft - Hochschule der Zukunft. Zukunft der Hochschulbildung - Future Higher Education. Wiesbaden: Springer VS. https://doi. org/10.1007/978-3-658-29297-3.

European Commission. (2020a). Commission staff working document. Accompanying the document: Communication from the commission to the european parliament, the council, the european economic and social committee and the committee of the regions. Digital education action plan 2021-2027. Resetting education and training for the digital age. Brüssel: European Commission. https://eur-lex.europa.eu/legal-content/DE/TXT/? uri=CELEX:52020SC0209. Zugegriffen: 30. Okt. 2020. 
European Commission. (2020b). European commission launches a public consultation on a new digital education action plan. https://ec.europa.eu/commission/presscorner/detail/en/ ip_20_1066. Zugegriffen: 30.Okt. 2020.

European Commission. (2020c). European universities initiative factsheet. Brüssel: European Union. https://ec.europa.eu/education/resources-and-tools/document-library/ european-universities-initiative-factsheet_en. Zugegriffen: 30. Okt. 2020.

European Commission, EACEA, \& Eurydice. (2018). The European higher education area in 2018: Bologna process implementation report. Luxemburg: Publications Office of the European Union. https://eacea.ec.europa.eu/national-policies/eurydice/content/europeanhigher-education-area-2018-bologna-process-implementation-report_en. Zugegriffen: 30. Okt. 2020.

European Commission, EACEA, \& Eurydice. (2020). The European higher education area in 2020: Bologna process implementation report. Luxemburg: Publications Office of the European Union. https://eacea.ec.europa.eu/national-policies/eurydice/content/europeanhigher-education-area-2020-bologna-process-implementation-report_en. Zugegriffen: 19. Nov. 2020.

Europäische Kommission. (2018). Mitteilung der Kommission an das Europäische Parlament, den Rat, den Europäischen Wirtschafts- und Sozialausschuss und den Ausschuss der Regionen zum Aktionsplan für digitale Bildung. Drucksache, 20/18. Berlin: Bundesrat. https://www.bundesrat.de/SharedDocs/drucksachen/2018/0001-0100/20-18.html. Zugegriffen: 30. Okt. 2020.

Europäische Kommission. (2020). Mitteilung der Kommission an das Europäische Parlament, den Rat, den Europäischen Wirtschafts- und Sozialausschuss und den Ausschuss der Regionen. Aktionsplan für digitale Bildung 2021-2027. Neuaufstellung des Bildungswesens für das digitale Zeitalter. Drucksache, 627/20. Berlin: Bundesrat. https://www. bundesrat.de/bv.html?id=0627-20. Zugegriffen: 30. Okt. 2020.

Forschung \& Lehre. (2020). 20 deutsche Hochschulen an neuen „EU-Universitäten “ beteiligt. Bonn: Deutscher Hochschulverband. https://www.forschung-und-lehre.de/politik/20deutsche-hochschulen-an-neuen-eu-universitaeten-beteiligt-2935/. Zugegriffen: 30. Okt. 2020 .

Gaebel, M., \& Zhang, T. (2018). Trends 2018: Learning and teaching in the european higher education area. Brüssel: EUA. https://eua.eu/resources/publications/757:trends-2018learning-and-teaching-in-the-european-higher-education-area.html. Zugegriffen: 30. Okt. 2020 .

Gemeinsame Erklärung der Europäischen Bildungsminister. (1999). Der Europäische Hochschulraum. Bologna. https://www.bmbf.de/files/bologna_deu.pdf. Zugegriffen: 30. Okt. 2020 .

Hackl, E. (2001). Towards a european area of higher education: Change and convergence in european higher education. EUI RSC 2001(9). Florenz: European University Institute. https://cadmus.eui.eu/handle/1814/1718. Zugegriffen: 30. Okt. 2020.

Harmsen, R., \& Deca, L. (2019). Taking stock of the bologna process at 20: The possibilities and limits of soft law governance. https://orbilu.uni.lu/handle/10993/39753. Zugegriffen: 30. Okt. 2020.

Hauschildt, K., Vögtle, E. M., \& Gwosć, C. (2018). EUROSTUDENT VI. Overview and selected findings. Bielefeld: wbv Media. https://www.eurostudent.eu/download_files/doc uments/EUROSTUDENT_VI_short_report.pdf. Zugegriffen: 30. Okt. 2020. 
Hochschulrektorenkonferenz (HRK). (Hrsg.). (2015). Standards und Leitlinien für die Qualitätssicherung im Europäischen Hochschulraum (ESG). Bonn: HRK. https://www.hrk.de/ uploads/media/ESG_German_and_English_2015.pdf. Zugegriffen: 30. Okt. 2020.

Huertas, E., Biscan, I., Ejsing, C., Kerber, L., Kozlowska, L., Marcos Ortega, S., \& Seppmann, G. (2018). Considerations for quality assurance of e-learning provision. Report from the ENQA working group VIII on quality assurance and e-learning. Occasional papers, 26. Brüssel: European Association for Quality Assurance in Higher Education AISBL. https://www.enqa.eu/publications/considerations-for-qa-of-e-learning-pro vision/. Zugegriffen: 30. Okt. 2020.

ICDE. (2019). Report of the ICDE working group on the present and future of Alternative Digital Credentials (ADC). Oslo: ICDE. https://www.icde.org/knowledge-hub/2019/4/10/ the-present-and-future-of-alternative-digital-credentials. Zugegriffen: 30. Okt. 2020.

Inamorato dos Santos, A., Punie, Y., \& Castaño-Muñoz, J. (2016). Opening up education: A support framework for higher education institutions. JRC Science for Policy Report. https://doi.org/10.2791/293408.

Kato, S., Galán-Muros, V., \& Weko, T. (2020). The emergence of alternative credentials. OECD Education Working Papers, 216. Paris: OECD Publishing. https://www.oecd-ili brary.org/education/the-emergence-of-alternative-credentials_b741f39e-en. Zugegriffen: 30. Okt. 2020.

Kercher, J., \& Plasa, T. (2020). Corona und die Folgen für die internationale Studierendenmobilität in Deutschland. Ergebnisse einer DAAD-Befragung von International Offices und Akademischen Auslandsämtern. Bonn: DAAD. https://static.daad.de/media/daad_de/ pdfs_nicht_barrierefrei/der-daad/analysen-studien/daad_2020_corona_und_die_folgen_ fuer_die_internationale_studierendenmobilitaet_in_deutschland.pdf. Zugegriffen: 30 . Okt. 2020.

Kerres, M. (2016). E-Learning vs. Digitalisierung der Bildung. Neues Label oder neues Paradigma? In: A. Hohenstein \& K. Wilbers (Hrsg.), Handbuch E-Learning. Köln: Fachverlag Deutscher Wirtschaftsdienst. https://learninglab.uni-due.de/sites/default/files/ele arning-vs-digitalisierung.pdf. Zugegriffen: 30. Okt. 2020.

KMK. (2016). Strategie der Kultusministerkonferenz „Bildung in der digitalen Welt“. https://www.kmk.org/themen/bildung-in-der-digitalen-welt/strategie-bildung-inder-digitalen-welt.html. Zugegriffen: 30. Okt. 2020.

KMK. (2019). Empfehlungen zur Digitalisierung in der Hochschullehre. Berlin: Sekretariat der Ständigen Konferenz der Kultusminister der Länder in der Bundesrepublik Deutschland. https://www.kmk.org/fileadmin/Dateien/veroeffentlichungen_beschluesse/ 2019/2019_03_14-Digitalisierung-Hochschullehre.pdf. Zugegriffen: 30. Okt. 2020.

Lübcke, M., \& Wannemacher, K. (2019). Bildungsverständnis im europaweiten Vergleich. Analyse von Konzeptionen und Narrativen von EU-Kommission und ausgewählter EULänder (Arbeitspapier, 49). Berlin: Hochschulforum Digitalisierung. https://doi.org/10. 5281/zenodo.4282353.

Miao, F., Mishra, S., Orr, D., \& Janssen, B. (2019). Guidelines on the development of open educational resources policies. Paris: UNESCO. https://www.unesco.de/sites/default/files/ 2020-01/Guidelines_on_the_Development_of_OER_Policies_2019.pdf.Zugegriffen: 30 . Okt. 2020. 
MICROBOL. (2020). Micro-credentials linked to the bologna key commitments. Desk research report. Brüssel: EUA. https://eua.eu/resources/publications/940:micro-credentialslinked-to-the-bologna-key-commitments.html. Zugegriffen: 30. Okt. 2020.

Orr, D., Pupinis, M., \& Kirdulyte, G. (2020). Towards a european approach for microcredentials: A study of practices and commonalities in offering micro-credentials in european higher education - Analytical report, NESET report. Luxemburg: Publications Office of the European Union.

Orr, D., Rampelt, F., \& Knoth, A. (2020). „Bologna Digital“ - Actively shaping the digital transformation in european higher education. In A. Curaj, L. Deca, \& R. Pricopie (Hrsg.), European higher education area. Challenges for a new decade (S. 583-596). Cham: Springer International Publishing. https://doi.org/10.1007/978-3-030-56316-5_36.

Orr, D., Rimini, M., \& van Damme, D. (2015). Open educational resources - A catalyst for innovation. Paris: OECD Publishing. https://www.oecd.org/innovation/open-educationalresources-9789264247543-en.htm. Zugegriffen: 30. Okt. 2020.

Paris Communiqué. (2018). https://www.ehea.info/media.ehea.info/file/2018_Paris/77/1/ EHEAParis2018_Communique_final_952771.pdf. Zugegriffen: 30. Okt. 2020.

Planells-Artigot, E., \& Moll-Lopez, S. (2020). Distant partners: A case study of global virtual teams between Spain and South Korea. In A. Curaj, L. Deca, \& R. Pricopie (Hrsg.), European higher education area. Challenges for a new decade (S. 517-530). Cham: Springer International Publishing.

Rampelt, F., Niedermeier, H., Röwert, R., Wallor, L., \& Berthold, C. (2018). Digital anerkannt. Möglichkeiten und Verfahren zur Anerkennung und Anrechnung von in MOOCs erworbenen Kompetenzen (2. Aufl.) (Arbeitspapier, 34). Berlin: Hochschulforum Digitalisierung. https://doi.org/10.5281/zenodo.1414388.

Rampelt, F., Orr, D., \& Knoth, A. (2019). Bologna Digital 2020. White Paper on Digitalisation in the European Higher Education Area. Berlin: Hochschulforum Digitalisierung. https:// doi.org/10.5281/zenodo.3484744.

Rampelt, F., \& Wagner, B. (2020). Digitalisierung in Studium und Lehre als strategische Chance für Hochschulen. Strategie-, Struktur- und Kulturentwicklung gestalten. In R. Stang \& A. Becker (Hrsg.), Zukunft Lernwelt Hochschule (S. 105-120). München: De Gruyter Saur. https://doi.org/10.1515/9783110653663-011.

Redecker, C. (2017). European framework for the digital competence of educators: DigCompEdu. Luxemburg: Publications Office of the European Union. https://ec.europa.eu/ jrc/en/publication/eur-scientific-and-technical-research-reports/european-framework-dig ital-competence-educators-digcompedu. Zugegriffen: 30. Okt. 2020.

Resei, C., Friedl, C., Staubitz, T., \& Rohloff, T. (2019). Micro-credentials in EU and global. CORSHIP. https://www.corship.eu/wp-content/uploads/2019/07/Corship-R1.1c_ micro-credentials.pdf. Zugegriffen: 30. Okt. 2020.

Rome Communiqué. (2020). https://www.ehea.info/Upload/Rome_Ministerial_Communi que.pdf. Zugegriffen: 19. Nov. 2020.

Souto-Otero, M. et al. (2019). Erasmus + higher education impact study. Final Report. Luxembourg: Publications Office of the European Union. http://sepie.es/doc/comunicac ion/publicaciones/2019/erasmus_higher_education_impact_study.pdf. Zugegriffen: 30 . Okt. 2020.

Szabó, M., \& Tück, C. (2018). Fostering Trust and Transparency Through Quality Assurance. In A. Curaj, L. Deca, \& R. Pricopie (Hrsg.), European Higher Education Area: The Impact 
of Past and Future Policies (S. 569-587). Cham: Springer International Publishing. https:// doi.org/10.1007/978-3-319-77407-7_34.

UNESCO. (2020). Quality assurance and recognition of distance higher education and TVET. UNESCO COVID-19 Education Response: Education Sector Issue Notes, 5.1. Paris: UNESCO. https://unevoc.unesco.org/home/UNEVOC+Publications/lang=en/akt= detail/qs=6387. Zugegriffen: 30. Okt. 2020.

Yerevan Communiqué. (2015). https://www.ehea.info/media.ehea.info/file/2015_Yerevan/ 70/7/YerevanCommuniqueFinal_613707.pdf. Zugegriffen: 30. Okt. 2020.

Open Access Dieses Kapitel wird unter der Creative Commons Namensnennung 4.0 International Lizenz (http://creativecommons.org/licenses/by/4.0/deed.de) veröffentlicht, welche die Nutzung, Vervielfältigung, Bearbeitung, Verbreitung und Wiedergabe in jeglichem Medium und Format erlaubt, sofern Sie den/die ursprünglichen Autor(en) und die Quelle ordnungsgemäß nennen, einen Link zur Creative Commons Lizenz beifügen und angeben, ob Änderungen vorgenommen wurden.

Die in diesem Kapitel enthaltenen Bilder und sonstiges Drittmaterial unterliegen ebenfalls der genannten Creative Commons Lizenz, sofern sich aus der Abbildungslegende nichts anderes ergibt. Sofern das betreffende Material nicht unter der genannten Creative Commons Lizenz steht und die betreffende Handlung nicht nach gesetzlichen Vorschriften erlaubt ist, ist für die oben aufgeführten Weiterverwendungen des Materials die Einwilligung des jeweiligen Rechteinhabers einzuholen. 